\title{
Transfer of molecular recognition information from DNA nanostructures to gold nanoparticles
}

Thomas G.W. Edwardson, Kai Lin Lau, Danny Bousmail, Christopher J. Serpell and Hanadi F. Sleiman*

Corresponding author: hanadi.sleiman@mcgill.ca

Department of Chemistry and Centre for Self-assembled Chemical Structures, McGill University, 801 Sherbrooke Street West, Montreal, QC H3A OB8, Canada 


\section{Abstract}

DNA nanotechnology offers unparalleled precision and programmability for the bottom-up organisation of materials. The approach relies on pre-assembling a DNA scaffold, typically containing hundreds of different strands, and using it to position functional components. A particularly attractive strategy is to employ DNA nanostructures not as permanent scaffolds, but as transient, reusable templates to transfer essential information to other materials. To our knowledge, this approach, akin to top-down lithography, has not been examined. Herein, we report a molecular printing strategy, which chemically transfers a discrete pattern of DNA strands from a 3D-DNA structure to a gold nanoparticle. We show that the particles inherit the DNA sequence configuration encoded in the parent template with highfidelity. This provides control over the number of DNA strands, their relative placement, directionality and sequence asymmetry. Importantly, the nanoparticles produced exhibit the site-specific addressability of DNA nanostructures, and are promising components for energy, information and biomedical applications.

\section{Introduction}

Since the power of DNA as a chaperone for the assembly of gold nanoparticles (AuNPs) was first exemplified, ${ }^{1,2}$ it has been successfully applied to the 1D, 2D and 3D organization of AuNPs. ${ }^{3,4}$ DNAmediated AuNP assembly typically employs poly-functionalized nanoparticles, and has yielded a range of functional AuNP superlattices. ${ }^{5-10}$ On the other hand, the construction of discrete nanoparticle assemblies of arbitrary geometry requires the use of DNA scaffolds to precisely position the nanoparticles. ${ }^{11-20}$ Often, complex DNA origami scaffolds are used to organize a few nanoparticles. ${ }^{21-25}$ Importantly, the information is solely provided by the scaffold, while the nanoparticle is either isotropically or mono-functionalized. DNA nanostructures have previously been employed as templates for the growth of nanowires and inorganic surface patterning. In these instances the overall shape of the structures is transferred to the substrate but the complex DNA sequence information encoded in the template is lost. ${ }^{26}$

Recently, the ability to impart particles themselves with the geometries and valences that we take for granted in molecular self-assembly has been proposed as an alternative approach, ${ }^{27,28}$ and it may open doors to higher levels of control over nanoparticle assembly with a minimum number of DNA strands. With this in mind, the creation of AuNPs which possess anisotropic DNA patterns has been highly sought after. ${ }^{28,29}$ For example, controlled spacing between two or three DNA strands on the AuNP surface has been achieved using 1D DNA templates. ${ }^{30-32}$ Microsphere clustering with in situ polymerization has been used to create DNA patches on polymer colloids. ${ }^{33}$ Surface immobilization has also been used, creating Janus particles with localized patches of DNA strands. ${ }^{34-37}$ The combination of surface techniques with a stepwise approach exploiting electrostatic repulsion has produced geometrically defined DNA-AuNPs with a controlled number of up to six strands. ${ }^{38}$ More recently, amphiphilic polymers have been used to allow regioselective DNA functionalization on gold nanoparticles. ${ }^{39}$ While these methods have produced 
AuNPs with a significant measure of anisotropic regioselectivity, the ability to position many DNA strands with different sequences into geometrically controlled patterns has remained elusive.

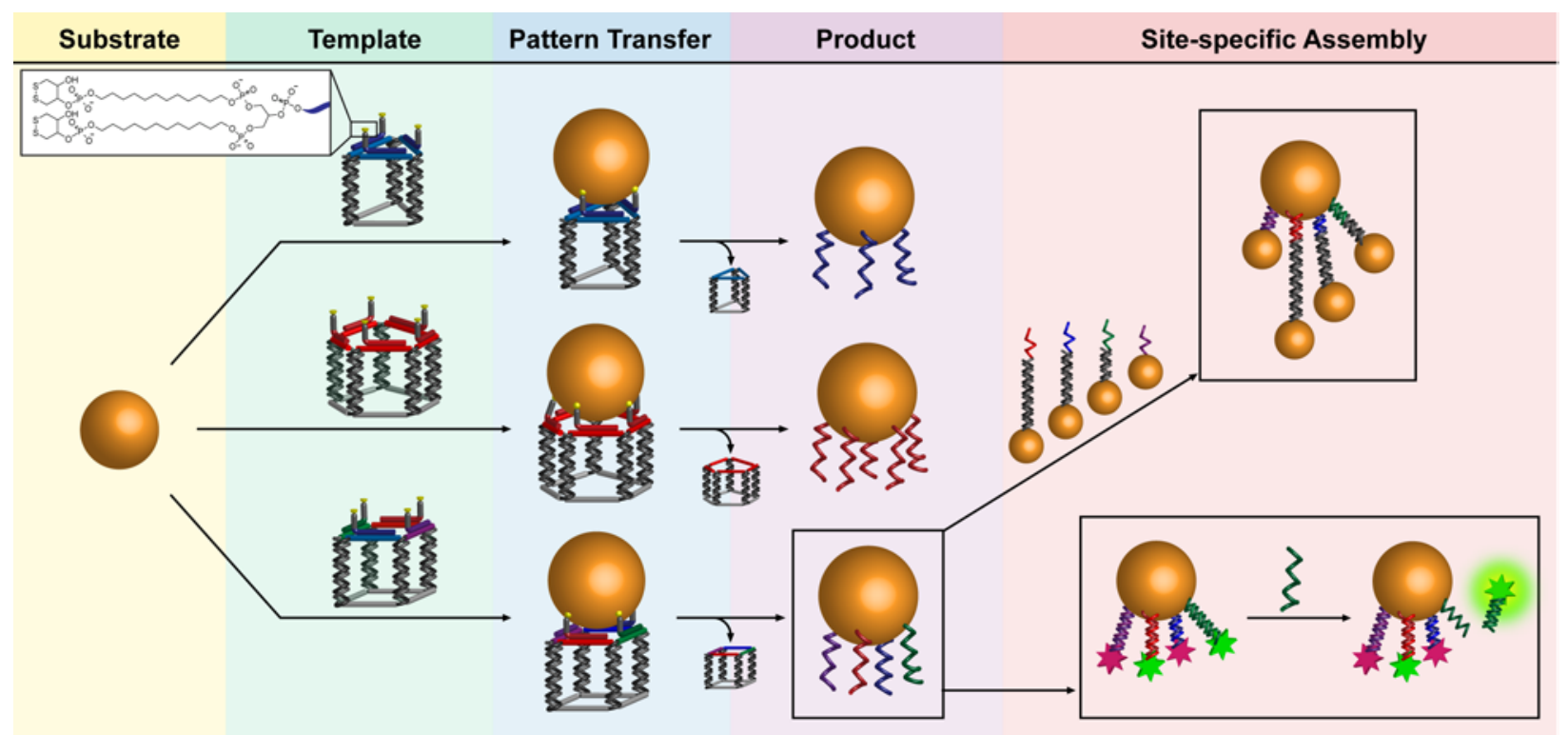

Figure 1 - An overview of the template-guided pattern transfer. An isotropic gold nanoparticle substrate is treated with a DNA nanostructure template, with controlled size, shape and sequence asymmetry (represented here as colors). The reactive arms presented by the DNA nanostructures bind covalently to gold nanoparticles, rendering a permanent pattern of DNA strands on the nanoparticle surface. The template structure is then removed, producing nanoparticles which have inherited molecular recognition information from the parent template. These information-rich nanoparticles are unique building blocks, which can be addressed site-specifically with different components, such as AuNPs with different spacer arms (top right) or different combinations of fluorescent probes (bottom right). They are also capable of dynamic assembly processes such as strand displacement (bottom right).

Herein, we describe a general method to transfer DNA strand motifs from a parent three-dimensional DNA template to gold nanoparticles, Figure 1. We show that the nanoparticle substrates acquire the DNA sequence information encoded in the parent template with high fidelity. Importantly, this produces nanoparticles which exhibit one of the defining properties of DNA nanostructures themselves: sitespecific addressability. To our knowledge, this represents the first example of the direct 'printing' of a DNA pattern onto gold nanoparticles from a 3D nanoscaffold, providing arbitrary control over the number of strands, their relative placement, directionality and sequence asymmetry. This creates directionally functionalized AuNPs, ${ }^{27}$ which are very promising components for the self-assembly of complex DNA programmed architectures. This also represents a first step in chemically copying the complex information contained in DNA nanostructures to another material, offering a potential approach to the problem of cost and scalability of DNA-based technologies. The process is related to the lithographic replication of electron beam patterns onto other materials, but here, the molecular information of the template is retained in the replication process. 


\section{Results and Discussion}

The goal of our approach is to produce a robust patterning of DNA strands on the nanoparticle surface. This requires anchoring groups appended to the oligonucleotide which can bind strongly to the gold surface and remain so after downstream processing and manipulation of the samples. ${ }^{29}$ With this in mind, a novel DNA-conjugate was synthesized, ${ }^{40,41}$ terminated with two cyclic disulfide moieties to allow AuNP-DNA conjugation (Supplementary S-IIla-f).

The bis-disulfide-DNA (Dx, where $x$ refers to the unique sequence) binds to AuNPs more efficiently and with faster binding kinetics than simple cyclic disulfide (CD) terminated DNA (Supplementary S-IIIh). The extending C12 portion in Dx may help to orient the disulfide moieties away from the highly charged DNA backbone, thus reducing electrostatic repulsion which is a key factor in the binding of DNA strands to gold nanoparticles. ${ }^{42}$ Furthemore, AuNPs functionalized with Dx were considerably more stable than the cyclic disulfide DNA (CD), as evidenced by a displacement assay using 1,4-dithiothreitol (DTT), ${ }^{43}$ see Supplementary S-IIli. These binding and stability data suggested that the Dx conjugates are well-suited to use in the patterning experiments to follow.
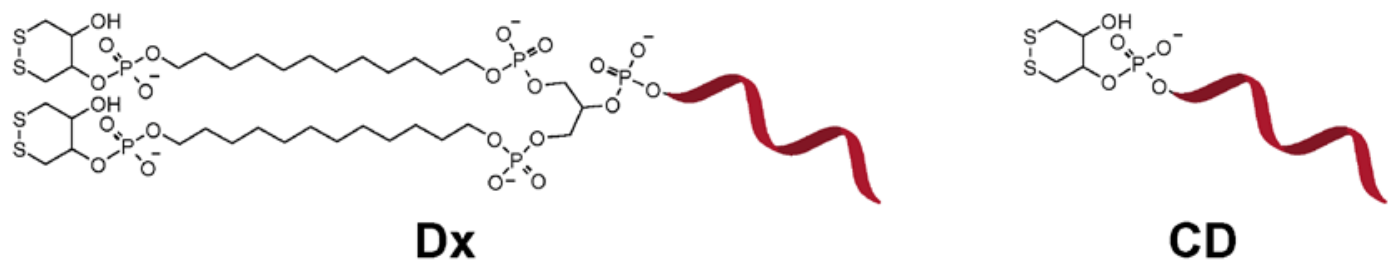

The criteria we have set for the DNA nanostructure used as a template are as follows; it has the potential for 1) geometric variation, 2) introduction of sequence asymmetry, 3) positioning of different numbers of Dx and 4) can be removed easily after pattern transfer. We have shown previously that DNA minimal 'clip-by-clip' based structures can be efficiently used to site-specifically position DNAamphiphiles and DNA-polymer conjugates. ${ }^{41,44,45}$ Here, the same strategy is employed to precisely arrange gold binding DNA conjugates for pattern transfer to a nanoparticle.

The first step in the patterning process is to prepare the template, in this case a 3D DNA scaffold decorated with Dx for transfer to the AuNP substrate. As such, the organization of different numbers of Dx on the DNA scaffold was carried out to produce a range of different letters for the printing process. (Figure 2a) The single-stranded regions of the cubic scaffold $\mathbf{C b}$ are 20 nucleotides (nt) in length. Based on previous designs, only the central 14nt were used for the binding of the Dx which have a spacer of $5 \mathrm{nt}$ to orient them away from the crowded corners of the structure. This design also lowers the melting temperature of this region to facilitate removal of the template after pattern transfer. The structures shown in Figure $2 \mathrm{~b}$ were assembled by one-pot thermal annealing of appropriate molar equivalents of 
the component DNA strands in a buffer solution containing $100 \mathrm{mM} \mathrm{Na}^{+}$. Quantitative yields of the desired products $\mathbf{C b}-\mathrm{Da}_{\mathbf{x}}(\mathrm{x}=1-4$, cubes with 1-4 $\mathrm{Da}$ strands positioned on one face) eliminated the need for purification of the DNA nanostructure prior to AuNP patterning. (Figure $2 b$ )

With the Da decorated cubic scaffolds $\mathbf{C b}-\mathrm{Da}_{\mathbf{x}}$ in hand, we proceeded to investigate their ability to bind to AuNPs and transfer the desired number of Da strands. Based on geometry calculations (Supplementary S-Va) 10nm AuNPs (NP ${ }^{10}$ ) were used, as they represent the best size match for the cubic scaffold. Gold nanoparticles were incubated with $\mathbf{C b}-\mathbf{D a}_{\mathbf{x}}$ at a 1:1 molar ratio for 16 hours at room temperature. The resulting hybrid DNA cage-nanoparticle assemblies, $\mathbf{N P}^{\mathbf{1 0}}-\mathbf{C b}-\mathbf{D} \mathbf{a}_{\mathbf{x}}$, (Figure $2 \mathrm{c}$ ) were analyzed by agarose gel electrophoresis (AGE) with reference to a control AuNP sample. (Figure 2d)

a

C
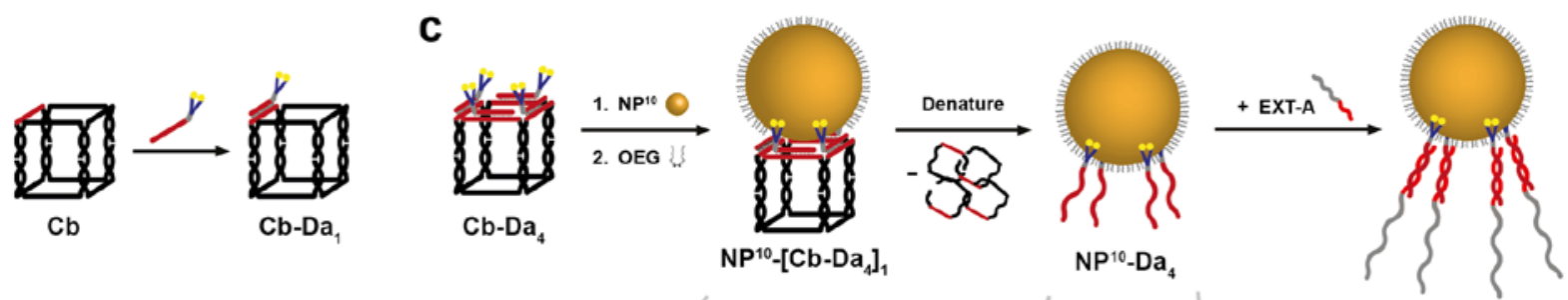

b
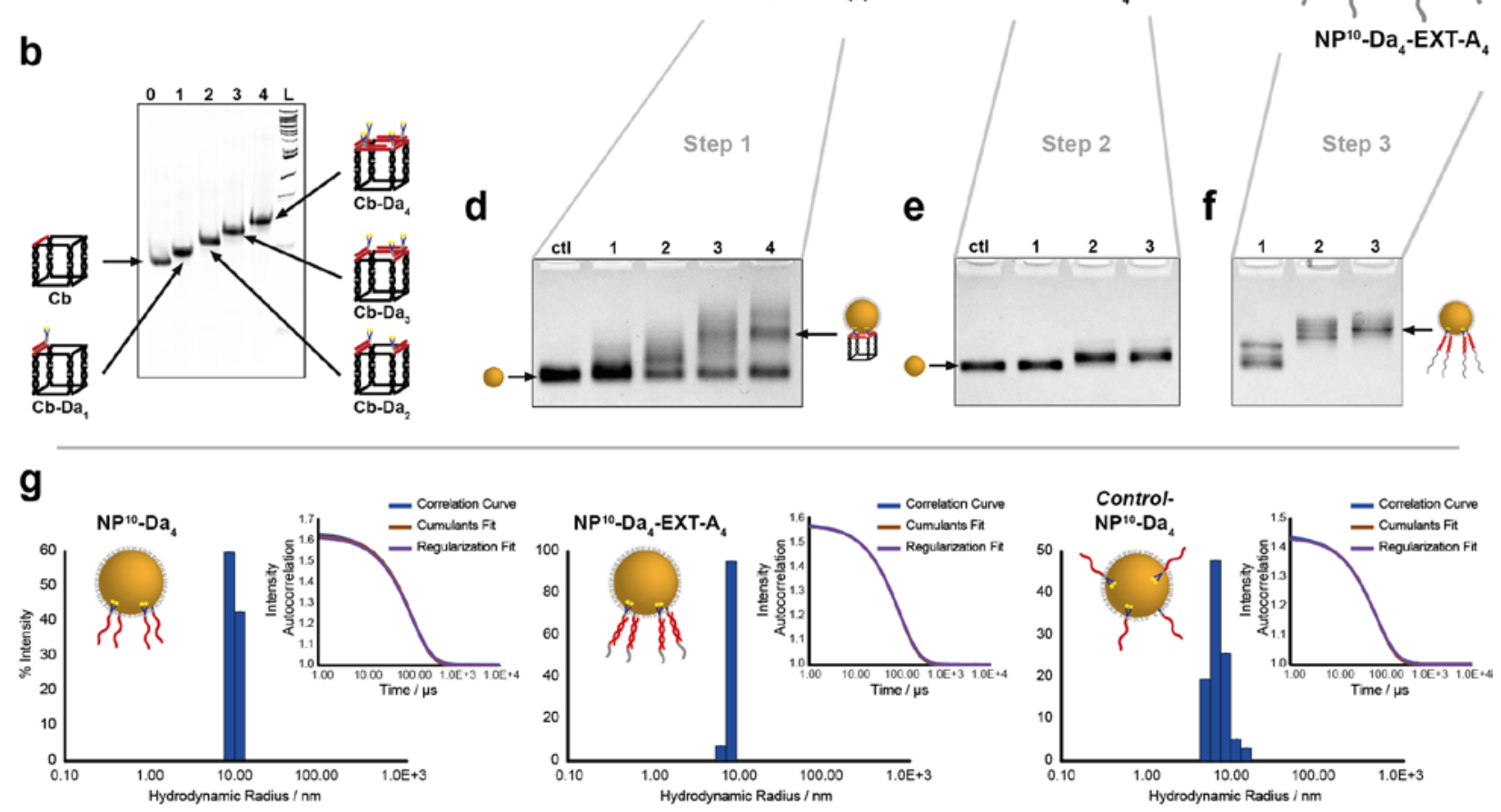

Figure 2 - Pattern transfer using cubic scaffold. a) The DNA cube, $\mathbf{C b}$, has eight ssDNA binding sites which can be addressed site specifically to organize Da molecules. b) Native Polyacrylamide Gel Electrophoresis (PAGE) analysis of Da addition to the cube

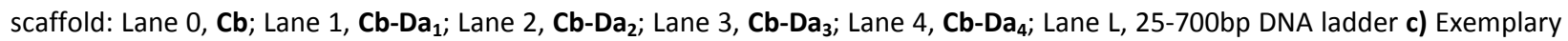
reaction scheme showing the binding of $\mathbf{C b}-\mathbf{D a}_{\mathbf{4}}$ to $\mathbf{N} \mathbf{P}^{\mathbf{1 0}}$ to produce the template bound $\mathbf{N} \mathbf{P}^{\mathbf{1 0}}-\left[\mathbf{C b}-\mathbf{D a}_{4}\right]$, which is treated with octaethylene glycol disulfide (OEG) for surface passivation before removal of the template by denaturing to produce $\mathbf{N P}^{\mathbf{1 0}}$-Da $\mathbf{a}_{4}$ which can then hybridize to four EXT-A strands to produce the structure $\mathbf{N P}^{\mathbf{1 0}}-\mathbf{D a}_{\mathbf{4}}-\mathbf{E X T}-\mathbf{A}_{\mathbf{4}}$ (drawn to approximate scale, see Supplementary S-Va for geometry calculations). d) AGE analysis of products obtained at step 1 for the $\mathbf{C b}_{-}-\mathbf{D a}_{\mathbf{x}}$ variants: Lane ctl,

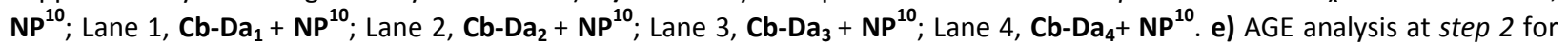

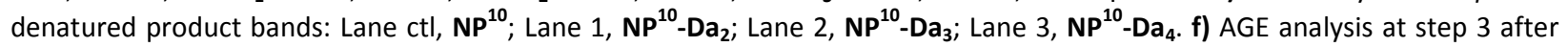

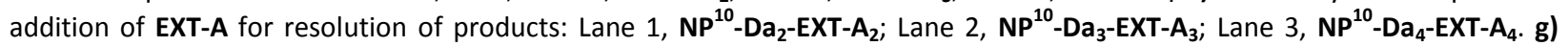


Dynamic light scattering (DLS) measurements of the templated product $\mathbf{N P}^{\mathbf{1 0}}-\mathbf{D a}_{\mathbf{4}}$ at steps 2 and 3 in comparison to a control sample in which four Da are positioned randomly on the nanoparticle reveals lower polydispersity, consistent with regioselectivity.

Electrophoretic mobility data for the reaction mixtures of cubes $\mathbf{C b}-\mathbf{D a}_{\mathbf{x}}$ with $10-\mathrm{nm}$ particles $\mathbf{N P}^{\mathbf{1 0}}$ (step 1) are shown in Figure $2 \mathrm{~d}$. While the binding of $\mathbf{C b}-\mathbf{D a}_{\mathbf{1}}$ and $\mathbf{C b}-\mathrm{Da}_{2}$ (cubes with one or two $\mathbf{D a}$ conjugates) to $\mathbf{N P}^{\mathbf{1 0}}$ is inefficient, both $\mathbf{C b}-\mathbf{D a}_{\mathbf{3}}$ and $\mathbf{C b}-\mathbf{D a}_{\mathbf{4}}$ show a product exhibiting mobility consistent with the $\mathbf{N P}^{10}-\left[\mathbf{C b}-\mathbf{D a}_{\mathbf{x}}\right]$ complex (see below for elucidation). Increasing conjugation yield is observed with increasing number of Da positioned on the cube, suggesting a cooperative effect due to the spatial organization on the template. (see below for the identity of the minor product of lower gel mobility in Figure 2d, lane 4) Control experiments using cubes without Dx strands revealed no interaction with AuNPs, confirming that binding was mediated by the Dx organized on the DNA cube (Supplementary Figure SF8).

To further stabilize the structures for downstream processing, passivation of the remaining surface of the AuNPs with a stable ligand was carried out. (Figure 2c) Carboxyl terminated octaethylene glycol disulfide (OEG) chains were used to cover the AuNP surface. Interestingly, after this stage the patterned AuNPs were found to be remarkably stable in sodium, and even in magnesium containing buffers. This passivation step could be carried out before or after removal of the template scaffold with no observable difference to the assemblies (see Supplementary Figure SF10). The samples were then run on AGE and the product bands excised and isolated by electroelution. The template scaffold was removed by disrupting all DNA hybridization under denaturing conditions (3M urea, 1XTBE), followed by centrifugation to isolate AuNPs. This process afforded purified $\mathbf{N} \mathbf{P}^{10}-\mathbf{D a} \mathbf{a}_{\mathbf{x}}$, see step 2 in Figure 2, with average isolated yields of $35 \%$ with respect to $\mathbf{N} \mathbf{P}^{\mathbf{1 0}}$. In principle, the DNA strand constituents of the cube may be isolated at this stage, and the cube re-assembled for repeated use as a transient template.

To determine the number of Dx strands transferred to the AuNP, and thus the fidelity of the patterning process, extension strands (EXT-X) were used to provide greater gel mobility differences, see step 3 in Figure 2. Importantly, these also probe the addressability of the Dx strands bound to the AuNP surface. The EXT-X strands have a $17 \mathrm{nt}$ region of complementarity with each Dx and an extending singlestranded DNA tail of 43 nt.

The gold nanoparticle containing 4 transferred DNA strands, $\mathbf{N P}^{10}-\mathbf{D a}_{\mathbf{4}}$ was incubated with an excess of EXT-A (Figure $2 f$, lane 3 ). The resulting sample exhibits decreased mobility, revealing that the Da strands transferred to the AuNP surface remain available for duplex formation and retain practical hybridization kinetics. The process was repeated for $\mathbf{N P}^{10}-\mathbf{D a}_{3}$ and $\mathbf{N} \mathbf{P}^{10}-\mathbf{D a}_{\mathbf{2}}$ in which the cube is bound to the nanoparticle via three or two DNA strands, and higher fidelity pattern transfer was observed with higher numbers of Da on the scaffold. In contrast, a non-templated control sample, prepared by incubating 4 molar equivalents of Da with $10 \mathrm{~nm}$ AuNPs displayed different behavior. Hybridization of this structure to EXT-A showed a statistical distribution of products with between one and four Da strands bound to the AuNP (Supplementary Figure SF9). Dynamic light scattering (DLS) analysis of $\mathbf{N P}^{\mathbf{1 0}}-\mathbf{D a}_{\mathbf{4}}$ showed a structure with less than $10 \%$ polydispersity, compared to the non-templated control with more than 
$20 \%$ polydispersity, corroborating the electrophoresis results and suggesting regioselective control via the cube template (see Supplementary S-Ve for further details).

DNA prismatic cages can be readily varied with respect to their geometry. Each geometry should transfer a different pattern of DNA strands to the nanoparticle, with different number and spacing of the Dx on the AuNP surface (Figure 3a). The preparation of Janus particles as a strategy to introduce anisotropy to spheres has been used to produce a range of asymmetrically functionalized AuNPs. ${ }^{34,35,37,39,46}$ Although hemispherical separation can be obtained, the number and placement of DNA strands on the AuNP is not precisely controlled.

To investigate this geometric control, triangular (TP) and pentagonal prism (PP) templates were synthesized in the same manner as for the cube, Figure $3 \mathrm{~b}$. The scaffolds were decorated with $\mathrm{Da}$ strands to produce TP-Da ${ }_{3}$ and PP-Da ${ }_{5}$ which were incubated with $10 \mathrm{~nm}$ AuNPs and then passivated with OEG. (Figure 3a)

a

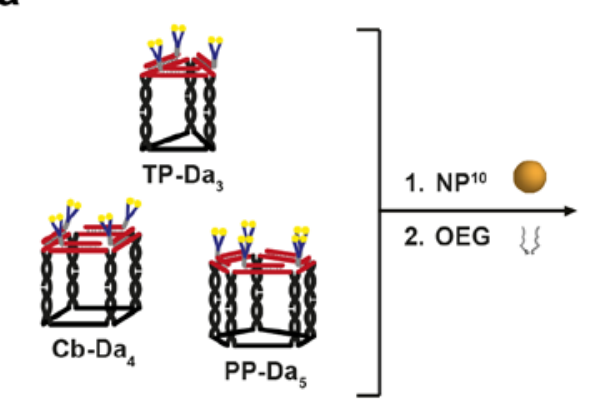

b
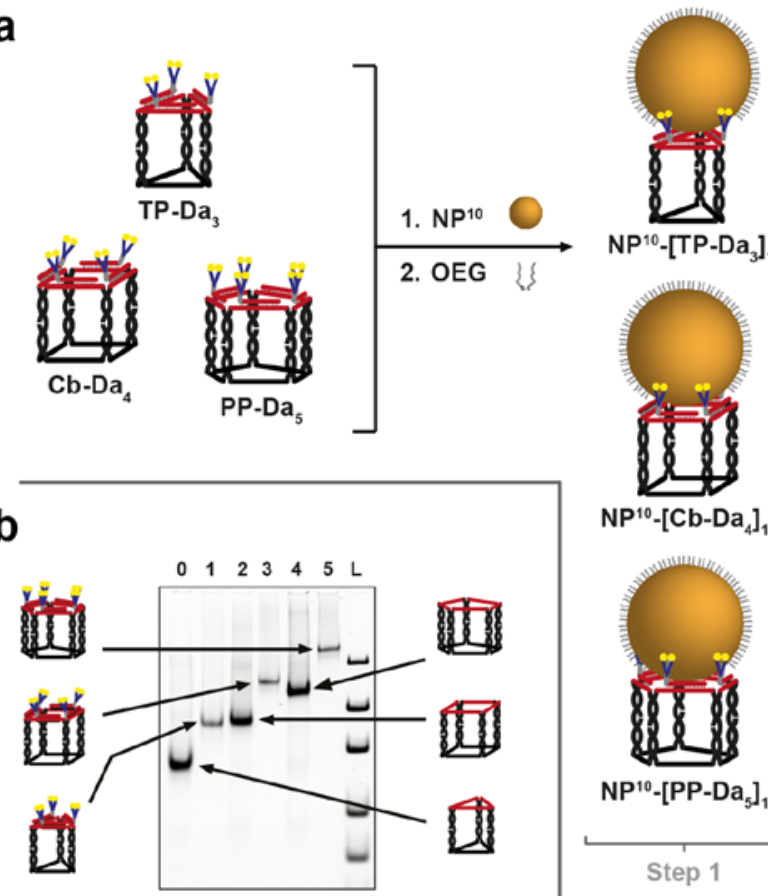

NP ${ }^{10}-\left[T P-\mathrm{Da}_{3}\right]_{1}$
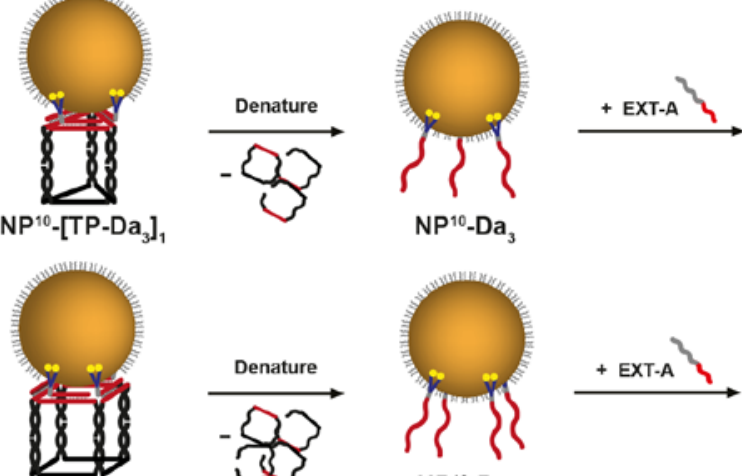

$N P^{10}-\left[\mathrm{Cb}-\mathrm{Da}_{4}\right]_{1}$

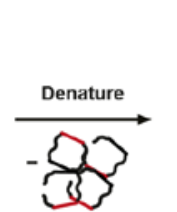

$\mathrm{NP}^{10}-\mathrm{Da}_{3}$
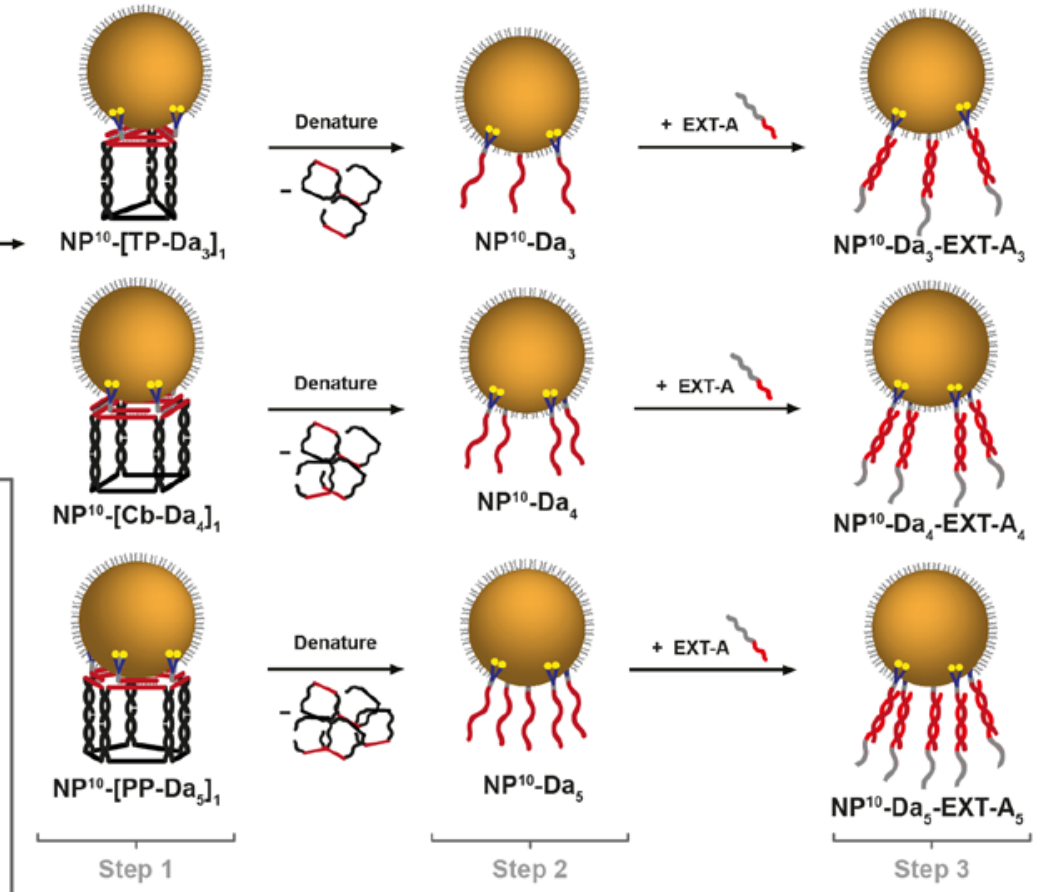

NP ${ }^{10}-\mathrm{Da}_{4}-\mathrm{EXT}-\mathrm{A}_{4}$

C

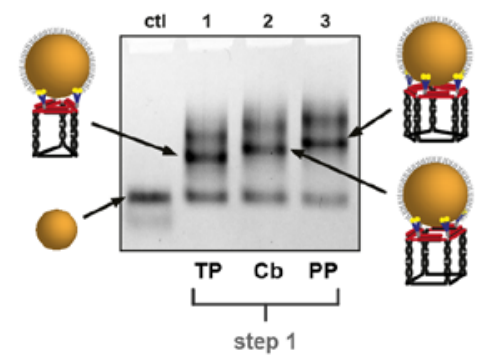

d

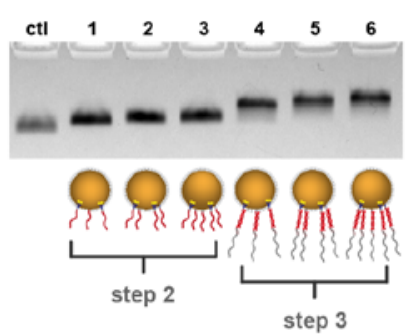

e

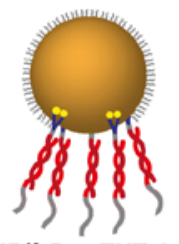

$\frac{\mathbf{N P}^{10}-\mathrm{Da}_{5}-\mathrm{EXT}^{-\mathbf{A}_{5}}}{\text { Step } 3}$

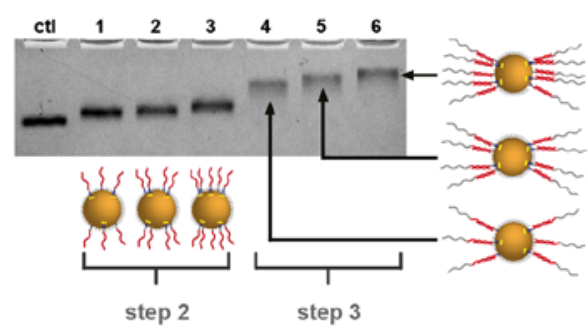


Figure 3 - Introduction of geometric diversity via template. a) General scheme for the patterning of AuNP using the DNA

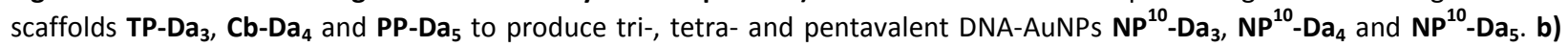
Native Polyacrylamide Gel Electrophoresis (PAGE) analysis of Da decorated prisms: Lane 0, TP; Lane 1, TP-Da; ; Lane 2, Cb; Lane

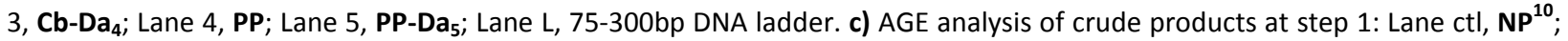

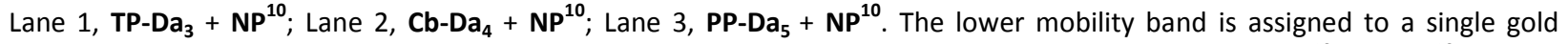
nanoparticle bound to two DNA prisms, see Figure 3e. d) AGE analysis of purified patterned products $\mathbf{N P}^{\mathbf{1 0}}-\mathbf{D a}_{3}, \mathbf{N P}^{\mathbf{1 0}}-\mathbf{D a}_{4}$ and $\mathbf{N P}^{10}-\mathbf{D a}_{5}$ and the addition of EXT-A strands: Lane ctl, NP ${ }^{10}$; Lane 1, NP ${ }^{10}-\mathbf{D a}_{3}$; Lane 2, NP ${ }^{10}-\mathbf{D a}_{4} ;$ Lane 3, NP ${ }^{10}-\mathbf{D a}_{5} . ;$ Lane $^{4}, \mathbf{N P}^{10}-$

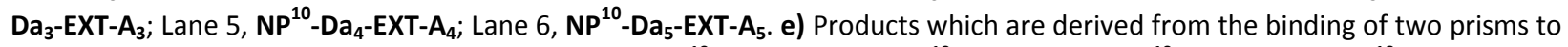
one AuNP, with and without EXT-A strands: Lane 0, NP ${ }^{10}$ (OEG); Lane 1, NP ${ }^{10}-\mathbf{D a}_{6}$; Lane 2, NP ${ }^{10}-\mathbf{D a}_{\mathbf{8}} ;$ Lane 3, NP ${ }^{10}-\mathbf{D a}_{10}$; Lane 4, NP $^{10}-$ Da $_{6}-$ EXT-A $_{6}$; Lane 5, NP ${ }^{10}-\mathrm{Da}_{8}-$ EXT-A $_{8}$; Lane 6, NP ${ }^{10}-$ Da $_{10}-$ EXT-A $_{10}$.

The prism-AuNP constructs were analyzed by AGE and a decrease in electrophoretic mobility was seen with increasing DNA scaffold size, consistent with the binding of the prisms to the AuNPs. In each case

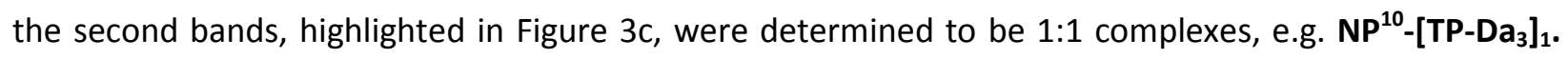
The band of lowest mobility was assigned as the 2:1 product, e.g. NP ${ }^{10}$-[TP-Da $]_{2}$, based on gel mobility and titration assays (Figure $3 e$ and Supplementary Figure SF11).

After purification and removal of the prism strands by denaturation, EXT-A was added to resolve the number of strands transferred to the AuNP. In Figure 3d, electrophoretic mobility patterns reveal that 3, 4 and 5 strands were robustly transferred by $\mathrm{TP}^{-\mathrm{Da}_{3}, \mathrm{C}-\mathrm{Da}_{4} \text { and PP-Da}} \mathbf{5}_{5}$, respectively. Additionally, hydrodynamic radii $\left(R_{h}\right)$ of the patterned products, determined by DLS, Supplementary S-Ve, revealed a steady increase with increasing number of strands on the nanoparticles, which is accentuated by the addition of extension strands EXT-A. Polydispersities were lower than non-templated gold nanoparticle conjugates (Supplementary Figures SF14-18 and Table ST5). These data, obtained for each step of the patterning process, demonstrate that well-defined numbers of DNA strands can be transferred to the AuNPs by geometric variation of the parent template.

Finally, we were interested in the potential of this strategy to transfer strands of different sequences to the nanoparticle. This confers particles with the ability for site-specific addressability and allows for anisotropic functionalization, thus producing building blocks with great potential for the self-assembly of advanced materials. ${ }^{27,28} \mathrm{~A}$ cubic scaffold with eight unique ssDNA binding sites Cb-Da-d was assembled and decorated with four unique gold binding conjugates; Da, Db, Dc, and Dd, on one face. (Figure 4a) Incubation with $10 \mathrm{~nm}$ AuNPs followed by OEG surface passivation, isolation of the major product, $\mathbf{N P}^{10}$. $[\mathbf{C b}-\mathrm{Da}-\mathbf{d}]_{1}$, and subsequent removal of the cubic scaffold, produced sequence asymmetric $\mathbf{N P}^{10}-\mathbf{D a}_{\mathbf{1}^{-}}$ $\mathbf{D b}_{1}-\mathbf{D c}_{1}-\mathbf{D d}_{1}$ (NP $\mathbf{P}^{10}$-Asym). To confirm the correct four strand DNA sequence code had been transferred to the AuNP, stepwise addition of four unique EXT strands, complementary to Da, Db, Dc and Dd (EXT-A to EXT-D) was carried out as before. In Figure $4 \mathrm{~b}$, the stepwise binding and site selective addressability of $\mathbf{N P}^{10}$-Asym is shown. In lane 3 the addition of EXT-A produces a particle with one extension strand bound and Db, Dc and Dd available for further hybridization, E1. The subsequent addition of the remaining three extension strands reveals a ladder of decreasing mobility as each additional EXT binds to its specific site on $\mathbf{N P}^{\mathbf{1 0}}$-Asym. This confirmed that the asymmetric pattern had been correctly transferred to the AuNP and the Dx are individually addressable due to their unique sequences. 
A control sample of AuNP incubated with one molar equivalent of each Dx strand (Da-Dd) without organization on a template was prepared under the same conditions as the patterned sample. This should produce a statistical mixture of number and identity of Dx on the AuNP. Stepwise addition of the EXT strands A-D reveals this in Figure 4c. This highlights the control over information transfer possible through the use of the DNA nanoscaffold template.

\section{a}

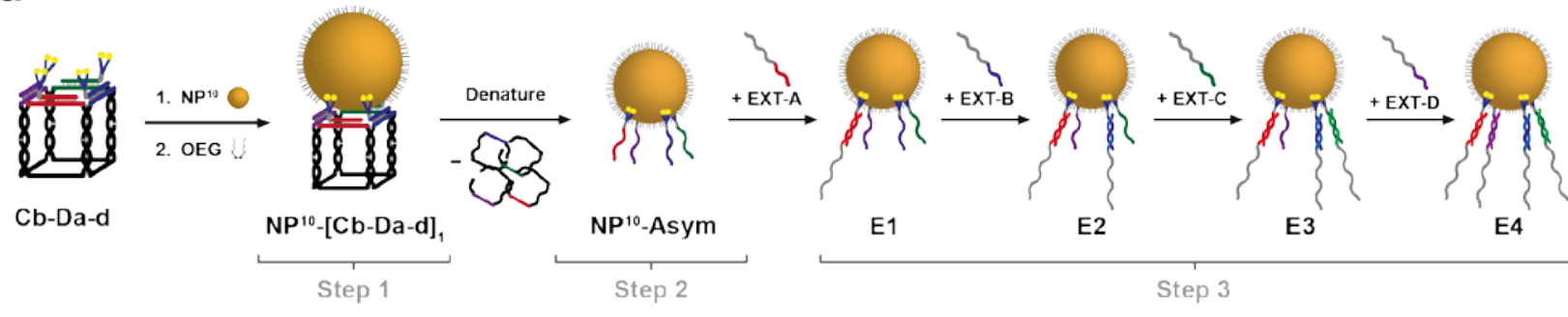

b

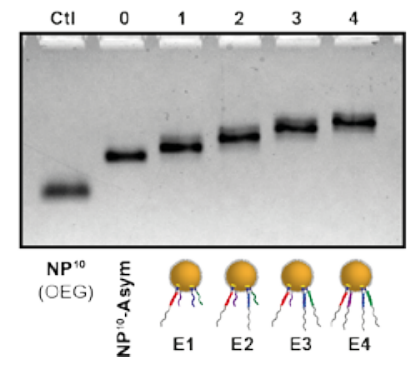

C

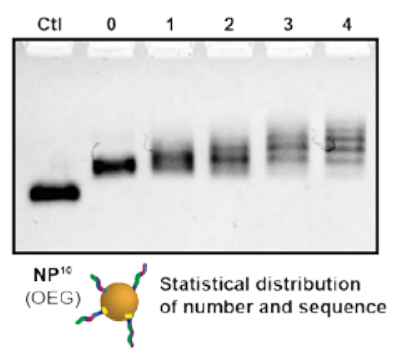

Figure 4 - Introduction of sequence asymmetry to patterning. a) General strategy for the production of sequence specifically patterned AuNPs using asymmetric DNA scaffolds Cb-Da-d to transfer one of each Dx strand to AuNP. After removal of cube template, each Dx strand on $\mathbf{N P}^{\mathbf{1 0}}$-Asym can be targeted individually to produce E1-4. b) AGE analysis of purified $\mathbf{N P}^{\mathbf{1 0}}$-Asym showing the site-specific binding of each unique EXT strand: Lane ctl, NP ${ }^{\mathbf{1 0}}$; Lane 0, NP ${ }^{\mathbf{1 0}}$-Da-d; Lane 1, E1; Lane 2, E2; Lane 3, E3.; Lane 4, E4 c) AGE analysis of template free control where one equivalent of each Da-Dd was incubated with $\mathbf{N P}^{\mathbf{1 0}}$, the addition of specific EXT strands reveals the statistical distribution of number and sequence: Lane ctl, NP ${ }^{10}(O E G)$; Lane 0, NP ${ }^{10}$ -

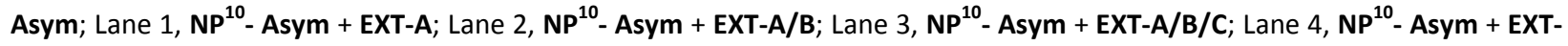
A/B/C/D.

To further characterize the asymmetrically functionalized nanoparticles, $\mathbf{N} \mathbf{P}^{\mathbf{1 0}}$-Asym, transmission electron microscopy (TEM) was used. Firstly, to visualize the asymmetric pattern and regioselectivity by TEM, polyvalent $6 n$ m AuNPs $\left(\mathbf{N P}^{6}\right)$ bearing complementary sequences $\left(\mathbf{a}^{\prime}-\mathbf{d}^{\prime}\right)$ to the patterned $\mathbf{N} \mathbf{P}^{\mathbf{1 0}}$ Asym were used as site-selective probes. The parent particle $\mathbf{N P}^{\mathbf{1 0}}$-Asym was incubated with an excess of $\mathbf{N P}^{6}-\mathbf{D} \mathbf{x}^{\prime}$, each with a sequence complementary to one of the strands on the nanoparticle (16 hours, room temperature, 1xTAMg) to produce the structures T1-T4 shown in Figure 5a. To aid microscopy analysis, assemblies were purified by AGE prior to TEM analysis, see Supplementary S-Vf. TEM revealed that the desired satellite structures were successfully generated, with yields decreasing for the more 
complex assemblies T3 and T4. The side products observed are likely due to the ability of the polyvalent particles to act as crosslinks between $\mathbf{N P}^{\mathbf{1 0}}$-Asym particles. Furthermore, inter-particle distances were consistent with the expected values for T1 and T2, however T3 and T4 showed mostly collapsed structures, possibly due to surface drying effects and $\mathrm{Mg}^{2+}$ mediated aggregation between the particles in such close proximity.

a

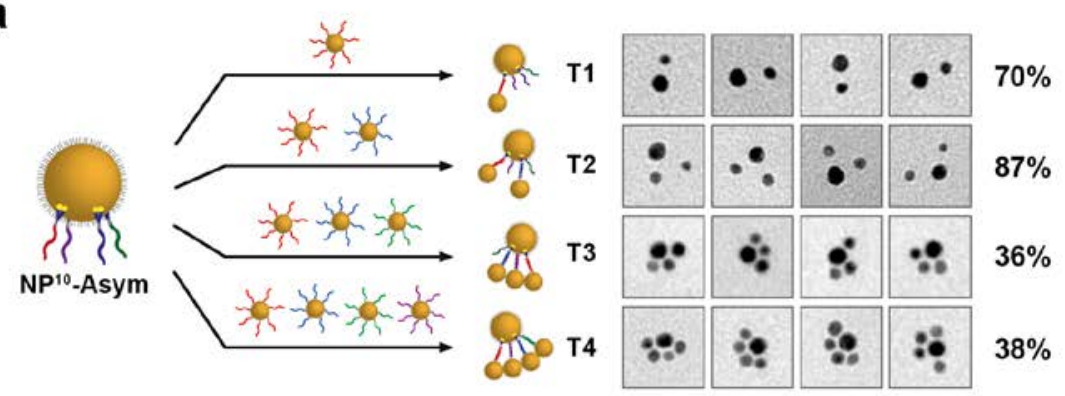

b

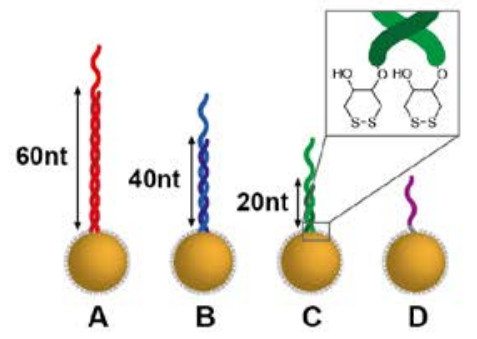

C

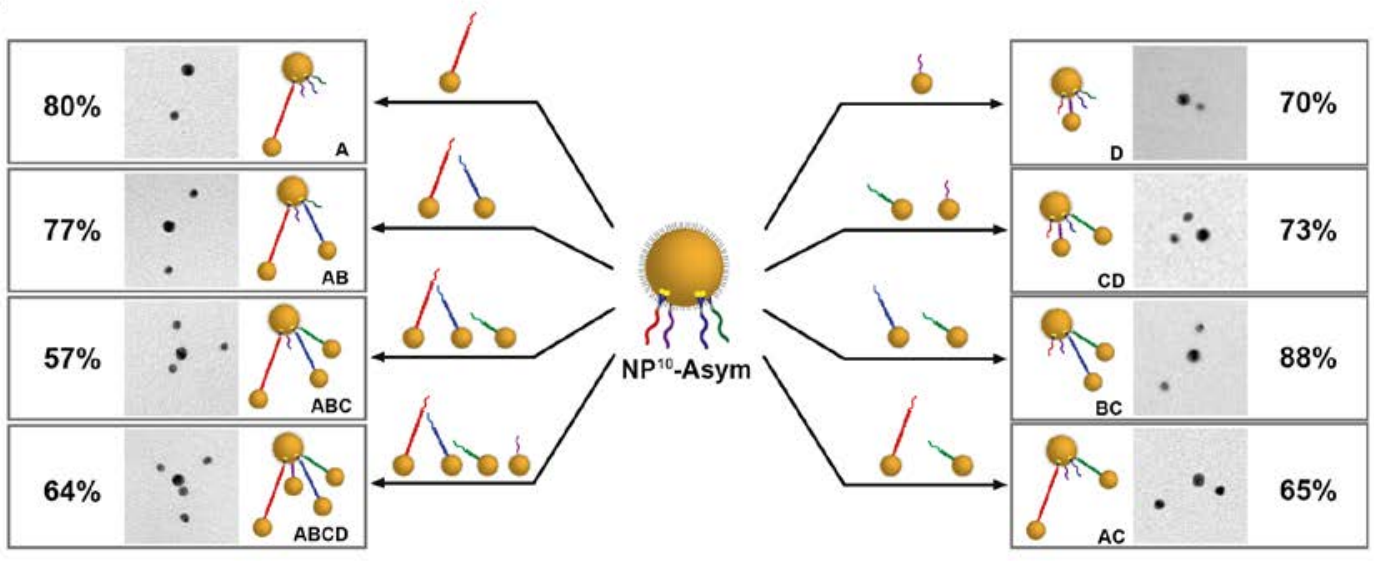

Figure 5 - Microscopy analysis of patterned AuNP self-assembly. a) TEM images show the site-specific addressability of the structure $\mathbf{N} \mathbf{P}^{10}$-Asym by hybridization of polyvalent $\mathbf{N P}^{6}$ (superscripts 10 and 6 are the nanoparticle diameters 10 and $6 \mathrm{~nm}$ ), labelled with complementary strands $\mathbf{D a}^{\prime}, \mathbf{D b}^{\prime}, \mathbf{D c}$ ' or $\mathbf{D d}$, the structure of these satellite structures shows the anisotropic nature of the patterning (for additional TEM images and statistics see supplementary S-Vg). b) Four different dsDNA arm lengths were created to allow each unique sequence (A-D) to be visualized via TEM. In each case the ssDNA binding portion is $17 \mathrm{nt}$ with sequences $\mathbf{A}, \mathbf{B}$ and $\mathbf{C}$ having dsDNA spacers of 20,40 and $60 \mathrm{bp}$ respectively. D has only the ssDNA $17 \mathrm{nt}$ binding region with no dsDNA portion. c) A range of anisotropic satellite structures can be accessed from the patterned particle $\mathbf{N} \mathbf{P}^{10}$ Asym and four unique monofunctionalized $\mathbf{N} \mathbf{P}^{5}$ with differing arm lengths $\mathbf{A}-\mathbf{D}$. This produces inter-particle separations of 30.4 , 23.6, 16.8 and $10.0 \mathrm{~nm}$ for $\mathbf{A}, \mathbf{B}, \mathbf{C}$ and $\mathbf{D}$ respectively. Additional experimental details and statistical analysis can be found in Supplementary S-Vf-h.

Although these assemblies highlight the sequence-specific addressability of the parent particle, $\mathbf{N P}^{\mathbf{1 0}}$ Asym, inherited from the asymmetric cube template, an improved design was developed to improve microscopy characterization. This uses rigid dsDNA arms of differing lengths on monofunctionalized nanoparticles to probe each addressable site on the $\mathbf{N P}^{\mathbf{1 0}}$-Asym. Complementary sequences to the four 
strands on the nanoparticles had different arm lengths, denoted A-D in Figure $5 \mathrm{~b}$. These should produce inter-particle distances of 30.4, 23.6, 16.8 and 10.0nm. Assemblies were carried out with an excess of the $5 \mathrm{~nm}$ monoconjugates A-D, and purified by AGE for TEM. Figure $5 \mathrm{c}$ shows representative images of some of the anisotropic satellite structures, which can be obtained from $\mathbf{N P}^{\mathbf{1 0}}$-Asym and the four unique arm lengths. These assemblies exhibited less collapsed structures and misassembled products than the structures T1-T4. This is consistent with the longer, more rigid, dsDNA arms distancing the particles from each other, and the DNA mono-conjugated nanoparticles. Additionally, inter-particle distances were measured for each of the assemblies and were found to correlate well with the expected values. A full statistical overview can be found in the Supplementary information, S-Vh. Overall, this method represents a simple, modular strategy to give access to many unique architectures using a small number of building blocks. More importantly, these experiments also reveal the potential of the information-rich patterned particles, $\mathbf{N P}^{10}$-Asym, to undergo selective DNA-mediated self-assembly, showcasing their viability for the production of unique DNA-AuNP assemblies.

To further investigate the sequence specificity imparted to the $\mathbf{N P}^{10}$-Asym particles a fluorescence assay was employed. A 'barcoded' particle with four different dye-labelled DNA strands was generated. Two fluorophores were used; 6-carboxyfluorescein (6-FAM) and cyanine 3 (Су3), which have discernible excitation/emission properties. We synthesized four 5' dye- labelled DNA sequences a' - d'; Cy3-a', Cy3b', 6-FAM-c' and 6-FAM-d'. Incubation of 1.5 molar equivalents of fluorophore to each unique binding site on $\mathbf{N P}^{10}$-Asym was carried out at room temperature for 12 hours. This was followed by three centrifugation and washing cycles, under native conditions, to remove any traces of unbound dyes.

a
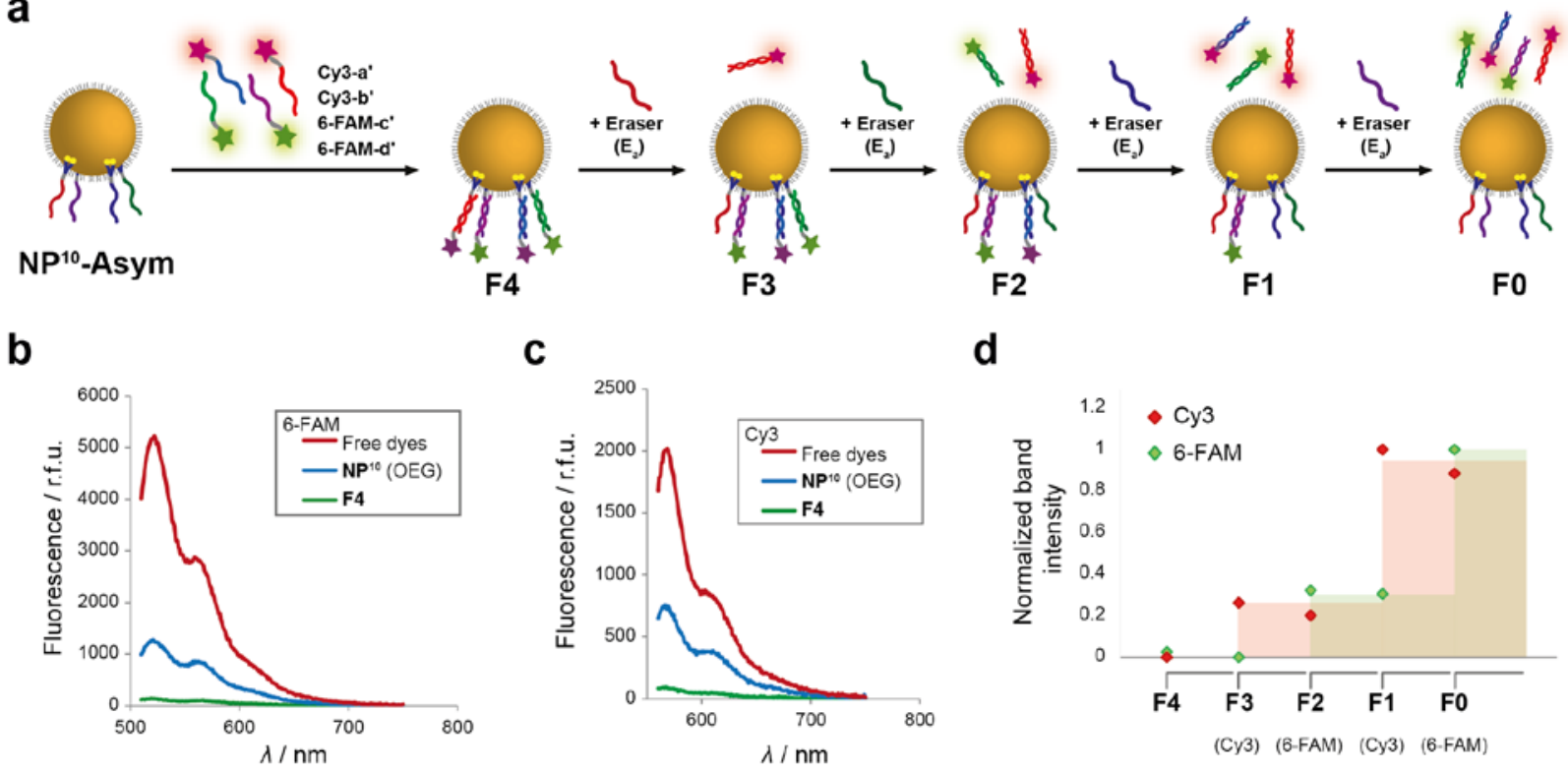

d

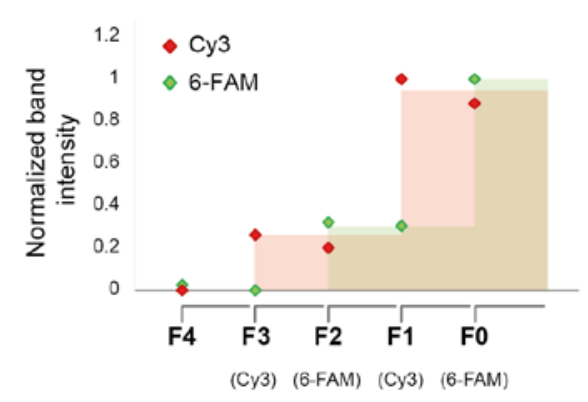

Figure 6 - Fluorescence investigation of asymmetric patterning. a) Scheme showing the preparation of a fluorophore 'barcode' on the sequence asymmetric $\mathbf{N P}^{\mathbf{1 0}}$-Asym using Cy3 and 6-FAM labelled oligonucleotides. b) Fluorescence emission spectra of 6FAM free in solution, in the presence of $\mathbf{N P}^{\mathbf{1 0}}(\mathrm{OEG})$ and when bound to the AuNP in structure F4 (Excitation; $490 \mathrm{~nm}$ ) $\mathbf{c}$ ) Spectra for $\mathrm{Cy} 3$ free in solution, in the presence of $\mathbf{N} \mathbf{P}^{10}(\mathrm{OEG})$ and when bound to the AuNP in structure $\mathbf{F} 4$ (Excitation; $545 \mathrm{~nm}$ ) $\mathbf{d}$ ) 
Histogram produced from AGE analysis of sequential strand displacement of dyes from $\mathbf{F} 4$ to finally produce F0. The order of eraser strands added was: $\mathbf{E}_{a}, \mathbf{E}_{c}, \mathbf{E}_{b}$ then $\mathbf{E}_{\mathrm{d}}$. This corresponds to alternating removal of the two dyes starting with $\mathbf{C y 3}$. The resulting increase in fluorescence at each step was seen to be dye specific, through the sequence specific eraser strands. (refer to Supplementary S-Vi for additional details).

Steady-state fluorescence spectra of the nanoparticle bearing all four dye-labelled DNAs, F4 in Figure 6a, were measured, and compared to control samples with the same concentration of free dyes in solution, and in the presence of unbound AuNP, NP ${ }^{10}(\mathbf{O E G})$. Firstly, a decrease in fluorescence is seen in the presence of $\mathbf{N P}^{10}(\mathbf{O E G})$ compared to the free dyes, suggesting some non-specific binding. For the sample F4, a large decrease in emission is observed when the dye-labelled DNAs are bound to the nanoparticle by hybridization to their surface bound complements. The fluorescence quenching observed is likely caused by both the AuNP as well as the close proximity of the dyes to each other. ${ }^{47}$

The efficient fluorescence quenching of the AuNP bound dyes allowed a displacement assay to be monitored directly. Four specific eraser strands, $\mathbf{E}_{\mathrm{a}}$ to $\mathbf{E}_{\mathrm{d}}$, were designed to remove the dye-tagged strands one-by-one from F4 by strand displacement, utilizing a 10nt toehold region for specificity and rapidity. Figure $6 \mathrm{~d}$ shows the cumulative addition of the different eraser strands, which will release the dyes from the AuNP sequentially, giving an increase in fluorescence emission of a specific dye at each step. For example, $\mathbf{E}_{\mathbf{a}}$ is added to $\mathbf{F} \mathbf{4}$ to liberate $\mathbf{C y 3}-\mathbf{a}$ ' and produce the structure $\mathbf{F 3}$. At this step an increase in Cy3 fluorescence is observed with negligible change in the 6-FAM fluorescence. The following displacement steps were carried out by alternating between the two dyes, in each case an increase in fluorescence for the specific displaced dye was seen. These results further support that four DNA strands with unique sequences have been transferred to the AuNP by the patterning method and present a platform with unique properties well-suited to biosensing. In particular, this method can result in a very large and diverse set of 'barcoded' gold nanoparticles from a minimum number of dyes of different colours, allowing efficient multiplexing and direct detection in bioanalyte sensing platforms. $^{48}$

\section{Conclusion}

In summary, we have developed a method to encode gold nanoparticles with complex DNA strand patterns comprising number, geometry and positioning of different unique strands. The use of DNA nanostructures as transient templates for the creation of patterned AuNPs is a general strategy which could be applied to a variety of inorganic nanoparticles. For example, the application of our approach to non-spherical particles could be used as a strategy for furthering complexity. This method also presents the advantage of potential recycling of the template or solid-support immobilization for high throughput, scalable generation of patterned structures. From a DNA nanotechnology standpoint this represents an interesting avenue to explore for the role of DNA scaffolds in nanofabrication, as a potential way to reduce costs by using DNA nanostructures in a transitory fashion. As well as allowing the transfer of different geometrical patterns to the AuNP, it is possible that the size ratio between the different DNA scaffolds and the AuNP could be used to further control the surface spacing and angle between DNA strands. In principle, the transfer should produce a chiral arrangement of the DNA strands 
on the gold nanoparticles, useful for the development of chiral plasmonic structures; we are currently working to test this stereospecificity.

As well as applications in colloidal assembly and biocomputing, ${ }^{49}$ we predict that this method could also be applied to surfaces to guide nanoelectronic or photonic circuitry or create robust anchors for positioning other functional components such as aptamers and enzymes. It is becoming well understood that surface structure and ligand placement are key factors to the cellular uptake of nanoparticles in vitro and their fate in vivo. This patterning creates a platform for exploring site-specific multivalent ligand-receptor or cell-surface interactions in combination with nucleic acid functionalized nanoparticles, for regulation of gene expression. ${ }^{50}$

\section{Methods}

\section{Preparation of DNA nanostructure patterned AuNPs.}

A generic protocol was developed and applied for all prism/sequence variants, here $\mathbf{C b}-\mathrm{Da}_{4}$ is provided as an example. In a typical patterning experiment, BSPP coated AuNPs (20 picomoles) were incubated with $\mathbf{C b}_{-} \mathbf{D a}_{4}(20$ pmol [3D construct]) (BSPP: bis(p-sulfonatophenyl)phenylphosphine). Final sample concentrations were ca. 300nM in a tris $(90 \mathrm{mM})$, boric acid $(90 \mathrm{mM})$, EDTA $(2 \mathrm{mM})$ and $\mathrm{NaCl}(100 \mathrm{mM})$ buffer (1xTBEN, pH 8.3) with $2 \mathrm{mM}$ BSPP. Following Incubation at room temperature overnight, 20,000 equivalents of OEG (ca. 0.2M in 1xTBEN) was added to stably passivate the surface. After a 30 minute incubation with OEG at room temperature, samples were centrifuged $(12000 \times \mathrm{g})$ at $4^{\circ} \mathrm{C}$, the supernatant was removed and fresh buffer added. This process was repeated at least twice before loading the samples on gel. Gel bands were assigned, see Figures 2 and 3 , and the desired band carefully excised. Samples were extracted by electroelution in 1XTBE buffer and subsequently concentrated by centrifugation $(15000 \times \mathrm{g})$ at $30^{\circ} \mathrm{C}$ and removal of supernatant. The 'pellet' was then washed with $3 \mathrm{M}$ urea in $0.625 \times$ TBE three times. This was followed by three washes with sterile de-ionized water. Finally, sample solutions were quantified by absorption spectroscopy, monitoring absorbance at 450nm. Further experimental details, protocols and characterization data can be found in the Supplementary Information.

\section{Ackowledgements}

The authors acknowledge the Natural Sciences and Engineering Research Council of Canada (NSERC), the Canada Foundation for Innovation (CFI), the Centre for Self-Assembled Chemical Structures (CSACS) and the Canada Research Chairs Program for financial support. T.G.W.E thanks the Canadian Institutes of Health Research (CIHR) for a Drug Development Training Program (DDTP) scholarship. D.B. thanks NSERC for a Bionano scholarship and C.J.S thanks NSERC for a Banting Postdoctoral Fellowship. H.F.S. is a Cottrell Scholar of the Research Corporation.

\section{Author Contributions}

H.F.S. and T.G.W.E. designed the project. T.G.W.E. primarily contributed to the production of experimental results. K.L.L. carried out gold nanoparticle synthesis, TEM analysis, preparation of pattered AuNPs for Fluorescence studies and aided in data interpretation. D.B. carried out all DLS 
experiments. C.J.S. synthesized clip strands $T C 3-A B, P C 4-A B$ and PC5-AB. All authors have agreed to all the content of the manuscript.

\section{Competing Financial Interests}

The authors declare no competing financial interests.

\section{References}

1 Mirkin, C. A., Letsinger, R. L., Mucic, R. C. \& Storhoff, J. J. 'A DNA-based method for rationally assembling nanoparticles into macroscopic materials'. Nature 382, 607-609 (1996).

2 Alivisatos, A. P. et al. 'Organization of 'nanocrystal molecules' using DNA'. Nature 382, 609-611 (1996).

3 Tan, S. J., Campolongo, M. J., Luo, D. \& Cheng, W. 'Building plasmonic nanostructures with DNA'. Nat Nano 6, 268-276 (2011).

4 Barrow, S. J., Funston, A. M., Wei, X. \& Mulvaney, P. 'DNA-directed self-assembly and optical properties of discrete 1D, 2D and 3D plasmonic structures'. Nano Today 8, 138-167 (2013).

5 Elghanian, R., Storhoff, J. J., Mucic, R. C., Letsinger, R. L. \& Mirkin, C. A. 'Selective Colorimetric Detection of Polynucleotides Based on the Distance-Dependent Optical Properties of Gold Nanoparticles'. Science 277, 1078-1081 (1997).

6 Park, S. Y. et al. 'DNA-programmable nanoparticle crystallization'. Nature 451, 553-556 (2008).

7 Nykypanchuk, D., Maye, M. M., van der Lelie, D. \& Gang, O. 'DNA-guided crystallization of colloidal nanoparticles'. Nature 451, 549-552 (2008).

8 Maye, M. M., Kumara, M. T., Nykypanchuk, D., Sherman, W. B. \& Gang, O. 'Switching binary states of nanoparticle superlattices and dimer clusters by DNA strands'. Nat Nano 5, 116-120 (2010).

9 Auyeung, E. et al. 'DNA-mediated nanoparticle crystallization into Wulff polyhedra'. Nature 505, 73-77 (2014).

10 Macfarlane, R. J., O'Brien, M. N., Petrosko, S. H. \& Mirkin, C. A. 'Nucleic Acid-Modified Nanostructures as Programmable Atom Equivalents: Forging a New "Table of Elements"'. Angewandte Chemie International Edition 52, 5688-5698 (2013).

11 Loweth, C. J., Caldwell, W. B., Peng, X., Alivisatos, A. P. \& Schultz, P. G. 'DNA-Based Assembly of Gold Nanocrystals'. Angewandte Chemie International Edition 38, 1808-1812 (1999).

12 Aldaye, F. A. \& Sleiman, H. F. 'Sequential self-assembly of a DNA hexagon as a template for the organization of gold nanoparticles'. Angewandte Chemie - International Edition 45, 2204-2209 (2006).

13 Aldaye, F. A. \& Sleiman, H. F. 'Dynamic DNA templates for discrete gold nanoparticle assemblies: Control of geometry, modularity, write/erase and structural switching'. Journal of the American Chemical Society 129, 4130-4131 (2007).

14 Li, H., Park, S. H., Reif, J. H., LaBean, T. H. \& Yan, H. 'DNA-Templated Self-Assembly of Protein and Nanoparticle Linear Arrays'. Journal of the American Chemical Society 126, 418-419 (2003).

15 Le, J. D. et al. 'DNA-Templated Self-Assembly of Metallic Nanocomponent Arrays on a Surface'. Nano Letters 4, 2343-2347 (2004).

16 Zhang, J., Liu, Y., Ke, Y. \& Yan, H. 'Periodic Square-Like Gold Nanoparticle Arrays Templated by Self-Assembled 2D DNA Nanogrids on a Surface'. Nano Letters 6, 248-251 (2006). 
Sharma, J. et al. 'Control of Self-Assembly of DNA Tubules Through Integration of Gold Nanoparticles'. Science 323, 112-116 (2009).

18 Chen, W. et al. 'Nanoparticle Superstructures Made by Polymerase Chain Reaction: Collective Interactions of Nanoparticles and a New Principle for Chiral Materials'. Nano Lett. 9, 2153-2159 (2009).

19 Mastroianni, A. J., Claridge, S. A. \& Alivisatos, A. P. 'Pyramidal and Chiral Groupings of Gold Nanocrystals Assembled Using DNA Scaffolds'. Journal of the American Chemical Society 131, 8455-8459 (2009).

Lau, K. L., Hamblin, G. D. \& Sleiman, H. F. 'Gold nanoparticle 3D-DNA building blocks: High purity preparation and use for modular access to nanoparticle assemblies'. Small 10, 660-666 (2014).

21 Ding, B. et al. 'Gold Nanoparticle Self-Similar Chain Structure Organized by DNA Origami'. Journal of the American Chemical Society 132, 3248-3249 (2010).

22 Shen, X. et al. 'Rolling Up Gold Nanoparticle-Dressed DNA Origami into Three-Dimensional Plasmonic Chiral Nanostructures'. Journal of the American Chemical Society 134, 146-149 (2011). Pal, S. et al. 'DNA Directed Self-Assembly of Anisotropic Plasmonic Nanostructures'. Journal of the American Chemical Society 133, 17606-17609 (2011).

24 Kuzyk, A. et al. 'DNA-based self-assembly of chiral plasmonic nanostructures with tailored optical response'. Nature 483, 311-314 (2012).

Shen, X. et al. 'Three-Dimensional Plasmonic Chiral Tetramers Assembled by DNA Origami'. Nano Letters 13, 2128-2133 (2013). Surwade, S. P. et al. 'Nanoscale Growth and Patterning of Inorganic Oxides Using DNA Nanostructure Templates'. Journal of the American Chemical Society 135, 6778-6781 (2013). Halverson, J. D. \& Tkachenko, A. V. 'DNA-programmed mesoscopic architecture'. Physical Review E - Statistical, Nonlinear, and Soft Matter Physics 87 (2013).

28 Kim, J.-W. \& Deaton, R. 'Molecular Self-Assembly of Multifunctional Nanoparticle Composites with Arbitrary Shapes and Functions: Challenges and Strategies'. Particle \& Particle Systems Characterization 30, 117-132 (2013). Zhang, T., Yang, Z. \& Liu, D. 'DNA discrete modified gold nanoparticles'. Nanoscale 3, 4015-4021 (2011).

30 Suzuki, K., Hosokawa, K. \& Maeda, M. 'Controlling the Number and Positions of Oligonucleotides on Gold Nanoparticle Surfaces'. Journal of the American Chemical Society 131, 7518-7519 (2009).

31 Zhang, T. et al. 'DNA Bimodified Gold Nanoparticles'. Langmuir : the ACS journal of surfaces and colloids 28, 1966-1970 (2011).

32 Zhang, C., Ma, J., Yang, J., Liu, S. \& Xu, J. 'Binding Assistance Triggering Attachments of Hairpin DNA onto Gold Nanoparticles'. Analytical Chemistry 85, 11973-11978 (2013). Wang, Y. et al. 'Colloids with valence and specific directional bonding'. Nature 491, 51-55 (2012). Xu, X., Rosi, N. L., Wang, Y., Huo, F. \& Mirkin, C. A. 'Asymmetric Functionalization of Gold Nanoparticles with Oligonucleotides'. Journal of the American Chemical Society 128, 9286-9287 (2006). encoding for high-throughput assembly of nanoclusters'. Nature materials 8, 388-391 (2009).

36 Feng, L., Dreyfus, R., Sha, R., Seeman, N. C. \& Chaikin, P. M. 'DNA Patchy Particles'. Advanced Materials 25, 2779-2783 (2013).

37 Xing, H. et al. 'DNA-Directed Assembly of Asymmetric Nanoclusters Using Janus Nanoparticles'. ACS Nano 6, 802-809 (2011).

38 Kim, J.-W., Kim, J.-H. \& Deaton, R. 'DNA-Linked Nanoparticle Building Blocks for Programmable Matter'. Angewandte Chemie International Edition 50, 9185-9190 (2011). 
39 Tan, L. H., Xing, H., Chen, H. \& Lu, Y. 'Facile and Efficient Preparation of Anisotropic DNAFunctionalized Gold Nanoparticles and Their Regioselective Assembly'. J. Am. Chem. Soc. 135, 17675-17678 (2013).

40 Carneiro, K. M. M., Aldaye, F. A. \& Sleiman, H. F. 'Long-range assembly of DNA into nanofibers and highly ordered networks using a block copolymer approach'. Journal of the American Chemical Society 132, 679-685 (2010).

41 Edwardson, T. G. W., Carneiro, K. M. M., McLaughlin, C. K., Serpell, C. J. \& Sleiman, H. F. 'Sitespecific positioning of dendritic alkyl chains on DNA cages enables their geometry-dependent self-assembly'. Nat Chem 5, 868-875 (2013).

42 Hurst, S. J., Lytton-Jean, A. K. R. \& Mirkin, C. A. 'Maximizing DNA Loading on a Range of Gold Nanoparticle Sizes'. Anal. Chem. 78, 8313-8318 (2006).

43 Li, Z., Jin, R., Mirkin, C. A. \& Letsinger, R. L. 'Multiple thiol-anchor capped DNA-gold nanoparticle conjugates'. Nucleic Acids Research 30, 1558-1562 (2002).

44 McLaughlin, C. K. et al. 'Three-dimensional organization of block copolymers on "DNA- minimal" scaffolds'. Journal of the American Chemical Society 134, 4280-4286 (2012).

45 Serpell, C. J., Edwardson, T. G. W., Chidchob, P., Carneiro, K. M. M. \& Sleiman, H. F. 'Precision Polymers and 3D DNA Nanostructures: Emergent Assemblies from New Parameter Space'. J. Am. Chem. Soc. 136, 15767-15774 (2014).

46 Huo, F., Lytton-Jean, A. K. R. \& Mirkin, C. A. 'Asymmetric Functionalization of Nanoparticles Based on Thermally Addressable DNA Interconnects'. Adv. Mater. (Weinheim, Ger.) 18, 23042306 (2006).

47 Reineck, P. et al. 'Distance and Wavelength Dependent Quenching of Molecular Fluorescence by Au@SiO2 Core-Shell Nanoparticles'. ACS Nano 7, 6636-6648 (2013).

48 De, M., Ghosh, P. S. \& Rotello, V. M. 'Applications of Nanoparticles in Biology'. Adv. Mater. (Weinheim, Ger.) 20, 4225-4241 (2008).

49 Nikitin, M. P., Shipunova, V. O., Deyev, S. M. \& Nikitin, P. I. 'Biocomputing based on particle disassembly'. Nat Nano 9, 716-722 (2014).

50 Rosi, N. L. et al. 'Oligonucleotide-modified gold nanoparticles for intracellular gene regulation'. Science 312, 1027-1030 (2006). 